\title{
A software tool that provides relevant information for diabetic patients to help prevent diabetic foot
}

\author{
${ }^{1}$ Shebin $\mathrm{S},{ }^{2}$ Mallikarjunaswamy $\mathrm{S}$ \\ ${ }^{I}$ Dept. of Computer Science \& Engineering, S.J.B Institute of Technology, Bangalore, India \\ ${ }^{2}$ Dept. of Computer Science \& Engineering, S.J.B Institute of Technology, Bangalore, India
}

\begin{abstract}
This project proposes a tool which can be very useful for diabetic patients for preventing diabetic foot. 347 million people worldwide have diabetes and 15\% of them have the chance of developing diabetic foot. This can result in Amputation of limbs. This can be prevented by educating patients about the dangers of developing diabetic foot. The software provides the patients with suggestions for their current condition.

The traditional way of managing diabetes and diabetic foot is to consult a doctor. But for people who are living in remote villages, where doctor is not available in the vicinity, it is difficult to consult the doctor in short intervals. The proposed tool will allow diabetic patients to get assistance without personally having to consult the doctor. So the proposed tool gives great advantage over the existing system.

Many of the existing systems such as clinical decision support systems (CDSS) provide the patient with diagnosis details only. Example for this is simulconsult, diagnosispro, visualdx etc. That means when a patient provides the symptoms to the tool, the tool will determine which disease that patient is having. The proposed system concentrates mainly in post diagnosis phase of diabetic patients in particular.

Moreover many other tools which exist currently provide the patient only with reminders. Reminders can be of the form of notification to take medicines which is prescribed to the diabetic patient. The proposed system differs itself by providing the diabetic patient with suggestions which is relevant to him or her. The suggestions are specific to the answer provided by the patient.

The tool also tries to identify situations where the attention of a doctor is required. It also gives the provision to make an appointment with a doctor. This allows the doctor to personally assist those patients whose conditions are critical. The less critical patients can use this tool to get the necessary suggestions and advices to manage their diabetes.

This tool can be use by either the patients who will get relevant suggestions according to the answers they have given for the test cases and it can be used by health professionals to provide new and useful information regarding the disease condition and update any new findings or information to help better serve the patient.
\end{abstract}

Keywords: Diabetic foot, Diabetes, Data Mining, Suggestion Based Meta Data Symptom Probabilistic Algorithm, Clinical Decision Support Systems.

\section{Introduction}

Diabetes is a condition where the amount of glucose is high which may be due to the inadequate production of insulin in the body. Insulin is a hormone which converts sugar and starch and other food into energy in the body. If a diabetic patient doesn't maintain a good lifestyle he can develop diabetic foot: this is a condition where the amount glucose is too much that amputation (cutting of limbs) is the only way for the patient to survive. There have been many initiatives in healthcare systems and professional circles and organizations around the world to raise the awareness of diabetic foot (DF). They have all agreed that amputation can be avoided if the patient is aware of his condition and if he exercises caution.

Not many papers address the problem of providing assistance to diabetic patients. But there is one paper which reviews electronic decision support tools for diabetes care-[1] "Review of Electronic Decision-Support Tools for Diabetes Care: A Viable Option for Low- and Middle-Income Countries?" by K. Mohammed Ali, S. Shah, N. Tandon. In this paper, the author list examples of electronic clinical reminders for improving quality of care in diabetes. This paper shows the importance of tools, which assist patients and indicates that such tools can indeed make positive improvements in patient; hence making this field of health care very productive and yielding result. Furthermore this paper deals with diabetes in general and doesn't specialize on diabetic foot. More over according to this paper, the existing tools lack user friendliness. Patients have said to experience frustration in working with the tools.

In another paper-[2] "A randomized trial of electronic clinical reminders to improve quality of care for diabetes and coronary artery disease" by T. D. Sequist, T.K. Gandhi, A.S. Karson, J.M. Fiskio, D. Bugbee, M. Sperling, E.F. Cook, E.J. Orav, D.G, Fairchild, D.W. Bates- the author tries to create an individualized electronic decision support and remainder to improve diabetes care in communities. Here only generalized reminders are given to the patients rather than specialized suggestions. Reminder may refer to notification to take prescribed 
medicines and to undergo tests suggested by the doctor. In the healthcare field specialized result is much more preferable than generalized results.

In the paper [3], "A controlled trial of web-based diabetes disease management: the MGH diabetes primary care improvement project", the outcomes of a web-based decision support tool is tested. The tool referred here is diabetes Disease Management Application (DMA). It was developed so that evidence-based management of type 2 diabetes can be improved. Through this paper, the author showed that Web-based patient-specific decision support can improve evidence-based parameters of diabetes care.

In the paper [4], "Web-based collaborative care for type 1 diabetes: a pilot randomized trial.", it is found if a Web-based diabetes case management program can make a positive impact on patients suffering from diabetes. Electronic medical record (EMR) is also used in this trial. The author showed that using a Web-based case management program can indeed be useful in keeping the patient's disease under check.

The proposed system tries to overcome the limitations of the existing system. The working of the proposed system is briefly described: In proposed system or the proposed tool the patient or a family person will answer a set of questions related to diabetic's test cases. The Result is rated by an automated system which rates each question and answer and generates a list of suggestions based on the answers. If it exceeds threshold set for the symptoms by an expert then an appointment is provided with the healthcare professionals. The algorithm used in this project is Suggestion Based Meta Data Symptom Probabilistic Algorithm.

Opposed to the existing system, the proposed system tries to provide specialized results to the user or patient. Specific means that the suggestion given will depend on the answer provided by the user or patient. The proposed system is also meant to be as user friendly as possible. In the existing system the users had experienced frustration in using the interface as mentioned in paper [1]. The proposed tool also tries to identify situations where the patient's condition is very critical and a doctor is required. The tool allows the doctor to concentrate on critical patients while the less critical patients are given proper advices and suggestions regarding their medical condition.

\section{Basic Concepts}

A suggestion Based Meta Data Symptom Probabilistic Algorithm is used in the proposed tool and it forms the basis of this paper. What this tool does is that it asks the patients a number of questions regarding their present condition. The tool collects all these information and applies the suggestion Based Meta Data Symptom Probabilistic Algorithm and gives suggestions to the patient as a result.

\section{Suggestion Based Meta Data Symptom Probabilistic Algorithm}

1. Data Set of the various answers from the screening tests is created for the user.

2. Each of answer is given a rating.

3. Aggregated rating is determined for each of the user.

4. If the rating falls in a given range then the person is given a different suggestions based on the diabetics symptoms he has. The various tablets and other functions changes based on this Probabilistic measure.

This is illustrated in the following diagram

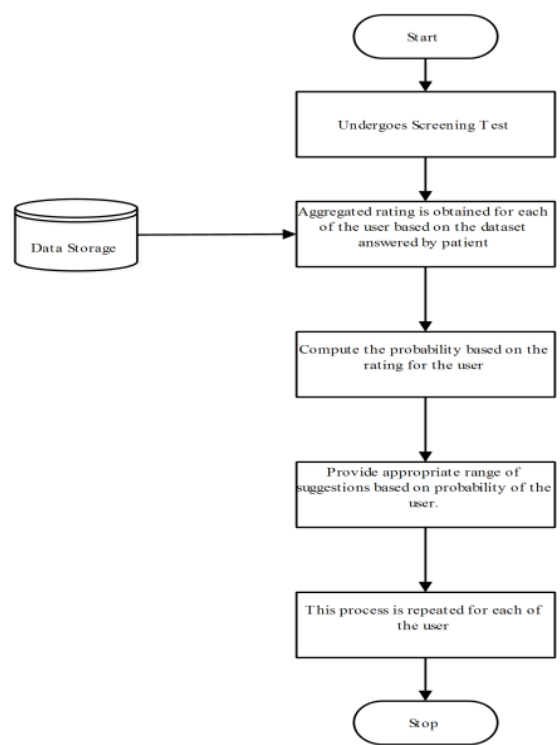

Fig.1. Suggestion Based Meta Data Symptom Probabilistic Algorithm 


\section{Description}

The traditional way how diabetic patients get assisted in prevention of diabetic foot is by consultation of doctor, information from internet and advices from friends and family. Consulting of doctor is the best way to deal with diabetic patient; but the problem is that a doctor may not be available 24 hours a day to advice the patient. The patient may visit the doctor once in a month but a doctor cannot give advices on a daily basis. Moreover, it is not really possible for a doctor to keep track of the minute details of the patient. Each patient will have a case file which includes the patient's medical history but sometimes the doctor may by mistake overlook some details. It is the minute details that may be non trivial in case of diabetes.

Patients also rely on information from the internet. There are so many websites and online journals that provide information regarding diabetes and diabetic foot. But the problem with that is that the information may be outdated or incorrect. This can make the problem worse. Not all patients are technology savvy to make use of internet facility also. Patients can ask for advice from family and friends. But in that case also false information can be obtained and can lead to more problems.

There are some clinical decision tools available that assist diabetic patients in general but there is no such tool that considers diabetic foot in specific. Most of the tools available only remind the patients about the various tests they have to undergo and very few of them provide patients with suggestions. The efficiency of most of these tools is not up to the level. Such tools have lots of drawbacks. These tools are not very user friendly according to reviews conducted by experts. Patients have said to have experienced frustration while working with these tools. Moreover, most of these clinical decision support systems (CDSS) are used for diagnosis purpose. These tools accept symptoms from the patient and perform certain operation on the input and give the diagnosis. These tools basically try to identify which disease the patient is having. The architecture of such a tool is shown in Fig 2.

The existing system only provides general suggestions to the patients. In health care field this is not recommended. Patients should get suggestions which are relevant to them personally. There is no one to one mapping between answers given by the patient and the suggestions because of which patient will not get relevant suggestions.

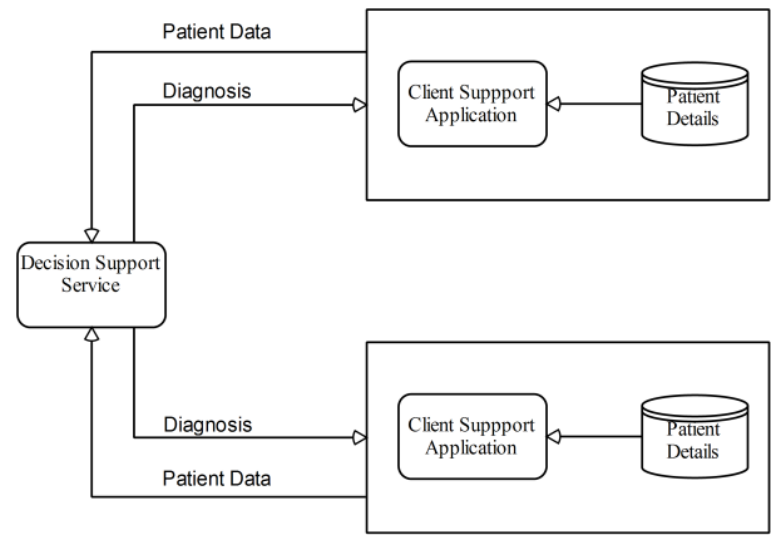

Fig.2. Existing system architecture overview

The proposed system is an application which will provide information to diabetic patients so as to prevent diabetic foot. It will act as a personalized tool for the patient. This tool can assist diabetic patients in leading a normal and healthy life. This allows doctors to find out the critical patient and give consultation to them. The less critical patients are managed by the proposed tool: they are given suggestions and advices according to their current medical condition. This tool is different in the sense that it concentrates on the post diagnosis stage of a patient. It is not a disease diagnosis tool; it is a tool to assist patients already suffering from diabetes.

The basic working of the proposed system is explained. The users or patients have to answer certain questions generated by the tool; based on these answers, the patient are classified as having low, medium or high diabetes. All the three categories of patients are given suggestion in accordance with the answer they have provided. The high diabetes patient refers to a patient whose condition is critical. So the tool provides option to make an appointment with the doctor. This is how the tool works. The architecture of the proposed tool is shown in Fig 3. 


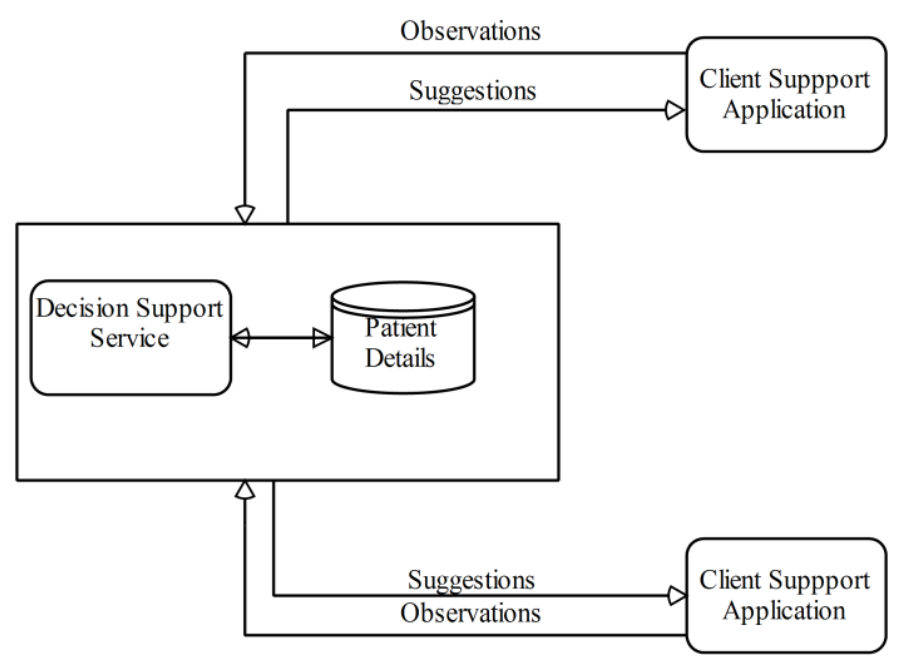

Fig.3. Proposed system architecture overview

\section{Comparison And Results}

The proposed system differs from the existing system in the fact that it deals with post diagnosis phase of a patient. The examples of the existing systems are SimulConsult, diagnosispro, visualdx and dxmate. In these tools the patient can input their observations or symptoms and the tool will predict which disease the patient is likely suffering. So basically the input is a group of symptoms and observation and output is the prediction regarding the disease suffered by the patients. These tools usually apply artificial intelligence algorithms on database containing symptom-disease information. Bayesian network, neural network, genetic Algorithms also may be used as the main algorithm in the existing system.

The proposed tool doesn't perform any diagnosis operation. It assumes that the patient is already suffering from diabetes. What the tool does is that it helps the patient in managing his medical condition. The patient has to answer a set of question which is generated by the tool. On the basis of those answers, the patient is given suggestions and advices. Example of suggestion may be to reduce intake of carbohydrate based food, do exercise for half an hour everyday etc. The advices and suggestion are generated using Suggestion Based Meta Data Symptom Probabilistic Algorithm. This is basically a logical condition technique.

There are similarities also between the existing and the proposed system. One such similarity is that both systems require a knowledge base to work on. In the existing system the knowledge base is provide by collecting symptom-disease information from doctors. In the proposed system, doctor has to create question and answers and give rating to each answer. Hence the common attribute is the requirement of a knowledge base.

As an end result the proposed tool met all its requirements: it successfully accepted the symptoms entered by the patient and provided the patient with advices and suggestions. A reasonable number of patients may use this tool at this time. This tool has helped in identifying diabetic patients whose conditions are critical or close to critical and have helped in gaining the attention of the doctor. So as a result a doctor may choose high priority patients first; with the help of the tool the low priority patients are also properly taken care of.

\section{Conclusion}

The proposed tool has successfully completed its objective. It can help the diabetic patients to manage their diabetes; the chance of having diabetic foot can be hence reduced. From the doctor's point of view, he/she will be able to identify the critical patients and can give immediate medical care to them. This tool really helps people who are living in remote locations where a doctor may not be available. The tool is made as user friendly as possible. The working of this tool is quite straight forward. As far as the patient is concerned, he/she only have to answer certain set of questions; which is quite straight forward.

The algorithm used here is Suggestion Based Meta Data Symptom Probabilistic Algorithm. In future work, this algorithm may be replaced by a better one if available. What is done in this paper is a unique approach to the problem of diabetes and diabetic foot. The algorithm may be modified if it results in more accurate results. The knowledge base of the tools can become rich with data by joining hands with multiple medical organizations. This tool even may be modified to deal with a different disease: it may be modified to assist cholesterol patients, hypertension etc. Overall, this paper tries to emphasis that technology can help humans live a healthier life. 


\section{References}

[1]. K. Mohammed Ali, S. Shah, N. Tandon, "Review of Electronic Decision-Support Tools for Diabetes Care: A Viable Option for Low- and Middle-Income Countries".

[2]. T. D. Sequist, T.K. Gandhi, A.S. Karson, J.M. Fiskio, D. Bugbee, M. Sperling, E.F. Cook, E.J. Orav, D.G, Fairchild, D.W. Bates, "A randomized trial of electronic clinical reminders to improve quality of care for diabetes and coronary artery disease".

[3]. Meigs JB, Cagliero E, Dubey A, Murphy-Sheehy P, Gildesgame C, Chueh H, Barry MJ, Singer DE, Nathan DM, “A controlled trial of web-based diabetes disease management: the MGH diabetes primary care improvement project", Diabetes Care. 2003;26(3), pp.750-757.

[4]. K.P. McCarrier, J.D. Ralston, I.B. Hirsch, G. Lewis, D.P. Martin, F.J. Zimmerman, H.I. Goldberg "Web-based collaborative care for type 1 diabetes: a pilot randomized trial. "2009;11(4),pp.211-217.

[5]. J.B. Meigs, E. Cagliero, A. Dubey, P. Murphy-Sheehy, C. Gildesgame, H. Chueh, M.J. Barry, D.E. Singer, D.M. Nathan, “A controlled trial of web-based diabetes disease management: the MGH diabetes primary care.

[6]. Holbrook, L. Thabane, K. Keshavjee, L. Dolovich, B.Bernstein B, D. Chan, S. Troyan , G. Foster, H.Gerstein, "Individualized electronic decision support and reminders to improve diabetes care in thecommunity: COMPETE II randomized trial”, CMAJ.09; 181(1-2), pp. 37-44

[7]. K.P. McCarrier, J.D. Ralston, I.B. Hirsch, G. Lewis, D.P. Martin, F.J. Zimmerman, H.I. Goldberg "Web based collaborative care for type 1 diabetes: a pilot randomized trial”, Diabetes technology and therapeutics 2009;11(4), pp. $211-217$. 